\title{
Comparison between the clot-protecting activity of a mutant plasminogen activator inhibitor-1 with a very long half-life and 6-aminocaproic acid
}

\author{
DANIEL GLENN KINDELL ${ }^{1}$, RICK WAYNE KECK ${ }^{1}$ and JERZY JANKUN ${ }^{1-3}$ \\ ${ }^{1}$ Urology Research Center, Department of Urology, University of Toledo, Health Science Campus, Toledo, OH 43614, USA; \\ ${ }^{2}$ Protein Research Chair, Department of Biochemistry, College of Sciences, King Saud University, \\ Riyadh 11451, Kingdom of Saudi Arabia; ${ }^{3}$ Department of Clinical Nutrition, \\ Medical University of Gdańsk, Gdańsk, Pomerania 80-211, Poland
}

Received August 20, 2014; Accepted November 17, 2014

DOI: $10.3892 /$ etm.2015.2399

\begin{abstract}
Plasminogen activator inhibitor (PAI)-1 is a serpin glycoprotein that can stabilize blood clots by inhibiting fibrinolysis. However, wild-type PAI-1 has the disadvantage of a short half-life of $\sim 2 \mathrm{~h}$. A very long half-life (VLHL) PAI-1 mutant was developed previously with an active-form half-life of $>700 \mathrm{~h}$, making it a possible candidate for use in hemorrhagic therapy. Current treatments for mitigating hemorrhage, other than inducers of blood clotting, are limited to lysine analog antifibrinolytics, including 6-aminocaproic acid and tranexamic acid. VLHL PAI-1 has been previously demonstrated to limit bleeding; however, the efficacy of this protein compared with lysine analog antifibrinolytics has not been investigated. The aim of the current study was to compare the clot stabilizing properties of the novel antifibrinolytic VLHL PAI-1 with those of 6-aminocaproic acid in reference plasma. Using thromboelastographic analysis, VLHL PAI-1 exhibited an $\mathrm{IC}_{50}$ (half maximal inhibitory concentration) of $8.8 \times 10^{-8} \mathrm{~mol} / \mathrm{l}$, while 6-aminocaproic acid showed an $\mathrm{IC}_{50}$ of $1.6 \times 10^{-4} \mathrm{~mol} / 1$. However, at doses of $>9.0 \times 10^{-7} \mathrm{~mol} / \mathrm{l}$, VLHL PAI-1 exhibited a delay in the onset of clot formation, which may be attributed to thrombin inhibition by excess PAI-1. The inhibition of tissue plasminogen activator by VLHL PAI-1 demonstrated improved efficacy over 6-aminocaproic acid in mitigating hemorrhage. In addition, patients with a PAI-1 deficiency, which causes blood clots to lyse rapidly resulting in profuse bleeding, may benefit from the application of VLHL PAI-1 as an antihemorrhagic therapy.
\end{abstract}

Correspondence to: Professor Jerzy Jankun, Urology Research Center, Department of Urology, University of Toledo, Health Science Campus, 3,000 Arlington Avenue, Toledo, OH 43614, USA

E-mail: jerzy.jankun@utoledo.edu

Key words: plasminogen activator inhibitor-1, 6-aminocaproic acid, thromboelastography, blood clotting

\section{Introduction}

Coagulation, the formation of a clot, is an essential process in the maintenance of homeostasis under normal conditions and during traumatic events. Numerous agents are used to initiate clotting and prevent uncontrolled bleeding. However, only a few agents, with limited efficacy, are used in clinical practice to protect formed clots against premature lysis $(1,2)$. The crosslinked polymer, fibrin, is dissolved into fibrin degradation products by plasmin. Thus, preventing the activation of plasmin is a potential target for antihemorrhage therapy. Plasmin, the primary fibrinolytic protease responsible for fibrin solvation, is formed by proteolytic cleavage of the zymogen plasminogen by tissue plasminogen activator (tPA) or urokinase plasminogen activator (uPA) (3-6). Vascular endothelial cells synthesize tPA, and tPA-mediated plasminogen activation is accelerated in the presence of fibrin $(5,6)$.

An appropriate level of plasminogen activation is maintained through the competing activity of plasminogen activators and plasminogen activator inhibitors (PAIs). The most relevant PAI in clot formation is PAI-1 (7), which is the quickest acting and most physiologically specific inhibitor of tPA $(5,8)$. The three conformational states of PAI-1 are latent (inactive), active and reactive-center-cleaved (plasminogen-activator complexed) (9). The active form of PAI-1 is rapidly converted (half-life, 1-2 h) into the latent form at physiological temperatures and $\mathrm{pH}(6,7,10)$. This relatively short half-life makes it difficult to use PAI-1 therapeutically as a clot protector. However, a previous study described a novel, genetically-engineered, antifibrinolytic PAI-1 with a significantly longer half-life $(>700 \mathrm{~h})$ compared with the wild-type (11).

The aim of the present study was to compare the antifibrinolytic activity of this very long half-life PAI-1 (VLHL PAI-1) with 6-aminocaproic acid (also known as $\varepsilon$-aminocaproic acid or Amicar) in the presence of tPA by establishing dose-response curves and determining the $\mathrm{IC}_{50}$ (half maximal inhibitory concentration) using thromboelastography (TEG). 
Table I. Selected thrombelastography parameters for the control-, VLHL PAI-1- and 6AC-treated reference plasma.

\begin{tabular}{|c|c|c|c|c|c|c|}
\hline Reagent $(\mu \mathrm{g})$ & $\mathrm{R}$ (min) & $\mathrm{K}(\min )$ & $\mathrm{A}\left({ }^{\circ}\right)$ & $\mathrm{MA}(\mathrm{mm})$ & $\mathrm{LY}_{30}(\%)$ & $\mathrm{G}\left(\mathrm{kdyn} / \mathrm{cm}^{2}\right)$ \\
\hline \multicolumn{7}{|l|}{ Control } \\
\hline Reference & $8.57 \pm 0.45$ & $2.04 \pm 0.62$ & $63.7 \pm 5.6$ & $26.2 \pm 2.2$ & $0.0 \pm 0.0$ & $1.77 \pm 0.19$ \\
\hline tPA-treated & $8.09 \pm 0.71$ & $2.30 \pm 0.55$ & $63.9 \pm 4.7$ & $19.3 \pm 2.5$ & $87.5 \pm 7.5$ & $1.21 \pm 0.19$ \\
\hline \multicolumn{7}{|l|}{ VLHL PAI-1 } \\
\hline 25.0 & $18.27 \pm 4.75$ & $5.80 \pm 1.80$ & $35.6 \pm 7.4$ & $30.2 \pm 1.5$ & $0.0 \pm 0.0$ & $2.17 \pm 0.11$ \\
\hline 12.5 & $23.75 \pm 0.70$ & $8.90 \pm 5.37$ & $32.1 \pm 13.2$ & $26.9 \pm 0.6$ & $0.0 \pm 0.0$ & $1.85 \pm 0.07$ \\
\hline 6.25 & $9.10 \pm 0.45$ & $2.67 \pm 1.55$ & $62.0 \pm 2.8$ & $26.4 \pm 0.6$ & $0.0 \pm 0.0$ & $1.82 \pm 0.35$ \\
\hline 3.12 & $8.70 \pm 0.28$ & $6.05 \pm 4.87$ & $65.0 \pm 13.1$ & $21.1 \pm 1.2$ & $0.0 \pm 0.0$ & $1.35 \pm 0.07$ \\
\hline 0.78 & $7.05 \pm 0.77$ & $2.30 \pm 1.55$ & $63.3 \pm 7.1$ & $24.8 \pm 4.5$ & $32.9 \pm 1.3$ & $1.70 \pm 0.42$ \\
\hline 0.39 & $8.00 \pm 0.98$ & $1.55 \pm 0.49$ & $71.2 \pm 2.4$ & $22.9 \pm 2.1$ & $85.0 \pm 2.3$ & $1.50 \pm 0.14$ \\
\hline 0.19 & $7.25 \pm 0.77$ & N/A & $62.4 \pm 4.8$ & $15.6 \pm 3.6$ & $91.8 \pm 0.5$ & $0.90 \pm 0.28$ \\
\hline \multicolumn{7}{|l|}{$6 \mathrm{AC}$} \\
\hline 36.0 & $8.20 \pm 0.00$ & $2.10 \pm 0.42$ & $63.3 \pm 8.4$ & $22.8 \pm 0.2$ & $0.0 \pm 0.0$ & $1.50 \pm 0.00$ \\
\hline 27.0 & $8.85 \pm 0.91$ & $5.10 \pm 2.68$ & $54.3 \pm 2.9$ & $21.1 \pm 1.4$ & $0.1 \pm 0.1$ & $1.35 \pm 0.07$ \\
\hline 18.0 & $8.20 \pm 0.40$ & $2.10 \pm 0.30$ & $67.0 \pm 2.8$ & $23.0 \pm 2.7$ & $2.4 \pm 2.3$ & $1.50 \pm 0.26$ \\
\hline 9.0 & $8.00 \pm 0.70$ & $1.60 \pm 0.28$ & $68.3 \pm 3.6$ & $24.0 \pm 0.6$ & $35.7 \pm 12.7$ & $1.55 \pm 0.07$ \\
\hline 4.5 & $8.85 \pm 1.20$ & $1.65 \pm 0.07$ & $67.3 \pm 1.6$ & $24.2 \pm 1.1$ & $48.3 \pm 1.1$ & $1.60 \pm 0.14$ \\
\hline 1.12 & $8.00 \pm 0.00$ & $1.80 \pm 0.00$ & $70.2 \pm 0.0$ & $21.8 \pm 0.0$ & $80.7 \pm 0.0$ & $1.40 \pm 0.00$ \\
\hline 0.75 & $8.55 \pm 0.07$ & $2.25 \pm 0.07$ & $62.9 \pm 2.4$ & $21.4 \pm 0.6$ & $87.1 \pm 0.3$ & $1.35 \pm 0.07$ \\
\hline
\end{tabular}

Values are expressed as the mean \pm standard deviation. $\mathrm{R}$, reaction time; $\mathrm{K}$, time from reaction until a 20 -mm clot formed; A, degree indicating the speed of clot formation; MA, maximum amplitude (maximum dynamic properties of platelet-fibrin interaction and contribution of fibrin to clot strength); G, clot strength (derived from MA); $\mathrm{LY}_{30}$, clot lysis $30 \mathrm{~min}$ after MA [detailed descriptions can be found in previous studies $(14,17,32)$ ]; VLHL PAI-1, very long half life plasminogen activator inhibitor-1; 6AC, 6-aminocaproic acid.

\section{Materials and methods}

Clot-protecting activity assay. A clot-protecting activity assay was performed using a TEG $^{\circledR} 5000$ Thrombelastograph ${ }^{\circledR}$ (Haemonetics Corporation, Braintree, MA, USA). The thrombelastograph measured coagulation with a pin attached to a torsion wire suspended in a cup holding a plasma sample. The $\mathrm{TEG}^{\circledR}$ was calibrated with plasma controls Level I (reading of TEG parameters within: R, 0-4 min; Angle, 75-87 MA, 42-63 mm; K, 0-2 min) and II (reading of TEG parameters within: R, 1-5 min; Angle, 60-80 ; MA, 26-41 mm; K, 0-6 min) (Haemoscope Corporation, Neils, IL, USA). Reference plasma (Coagulation Specialty Assayed Reference Plasma; Helena Laboratories Corporation, Beaumont, TX, USA) was reconstituted in $1 \mathrm{ml}$ distilled water and mixed with $20 \mu \mathrm{l}$ kaolin (Haemonetics Corporation). In trials where tPA was utilized, $20 \mu \mathrm{l}$ tPA ( $2 \mathrm{mg} / \mathrm{ml}$; Molecular Innovations, Novi, MI, USA) was added directly to the plasma. Next, $320 \mu \mathrm{l}$ plasma/kaolin solution was added to the $\mathrm{TEG}^{\circledR}$ cup containing $20 \mu \mathrm{l}$ calcium chloride $(0.2 \mathrm{M})$.

Trials. Each trial consisted of two samples running simultaneously, containing the same plasma/kaolin solution. The control $\mathrm{TEG}^{\circledR}$ cup received $10 \mu \mathrm{l}$ distilled water and the experimental $\mathrm{TEG}^{\circledR}$ cup received the appropriate combination of VLHL PAI-1, 6-aminocaproic acid (Sigma-Aldrich, St. Louis, MI, USA) and distilled water. The 6-aminocaproic acid was reconstituted with distilled water. Expression and purification of VLHL PAI-1 was conducted as described in a previous study (6). Serial dilutions of VLHL PAI-1 were performed with saline $(0.15 \mathrm{M})$ and each trial ran for $45 \mathrm{~min}$ at $37^{\circ} \mathrm{C}$.

Statistical analysis. Statistical analysis was performed using GraphPad Prism (GraphPad Software, Inc., La Jolla, CA, USA), and differences between the groups were analyzed by two-way analysis of variance. The data were presented as the mean \pm standard deviation, and $\mathrm{P}<0.05$ was considered to indicate a statistically significant difference. Graph visualization was performed using Origin 8 software (OriginLab, Northampton, MA, USA).

\section{Results}

Clotting parameters for the reference and $P$ PA-treated plasma. Selected clotting parameters for the control and various doses of 6-aminocaproic acid and VLHL PAI-1 are shown in Table I and Figs. 1 and 2. Control samples consisted of reference and tPA-treated plasma, with tPA included to mimic clot lysis. Reference and tPA-treated plasma exhibited similar average control values, with the exception of a lower clot strength $(\mathrm{G})$, maximum amplitude (MA) and rate of clot lysis after $30 \mathrm{~min}$ $\left(\mathrm{LY}_{30}\right)$ in the tPA-treated plasma due to tPA-induced clot lysis, which is consistent with previous studies (12-16). The MA was lower, as reference plasma lacks platelets that strengthen the blood clot $(3,17-19)$. There are no normal values of TEG 

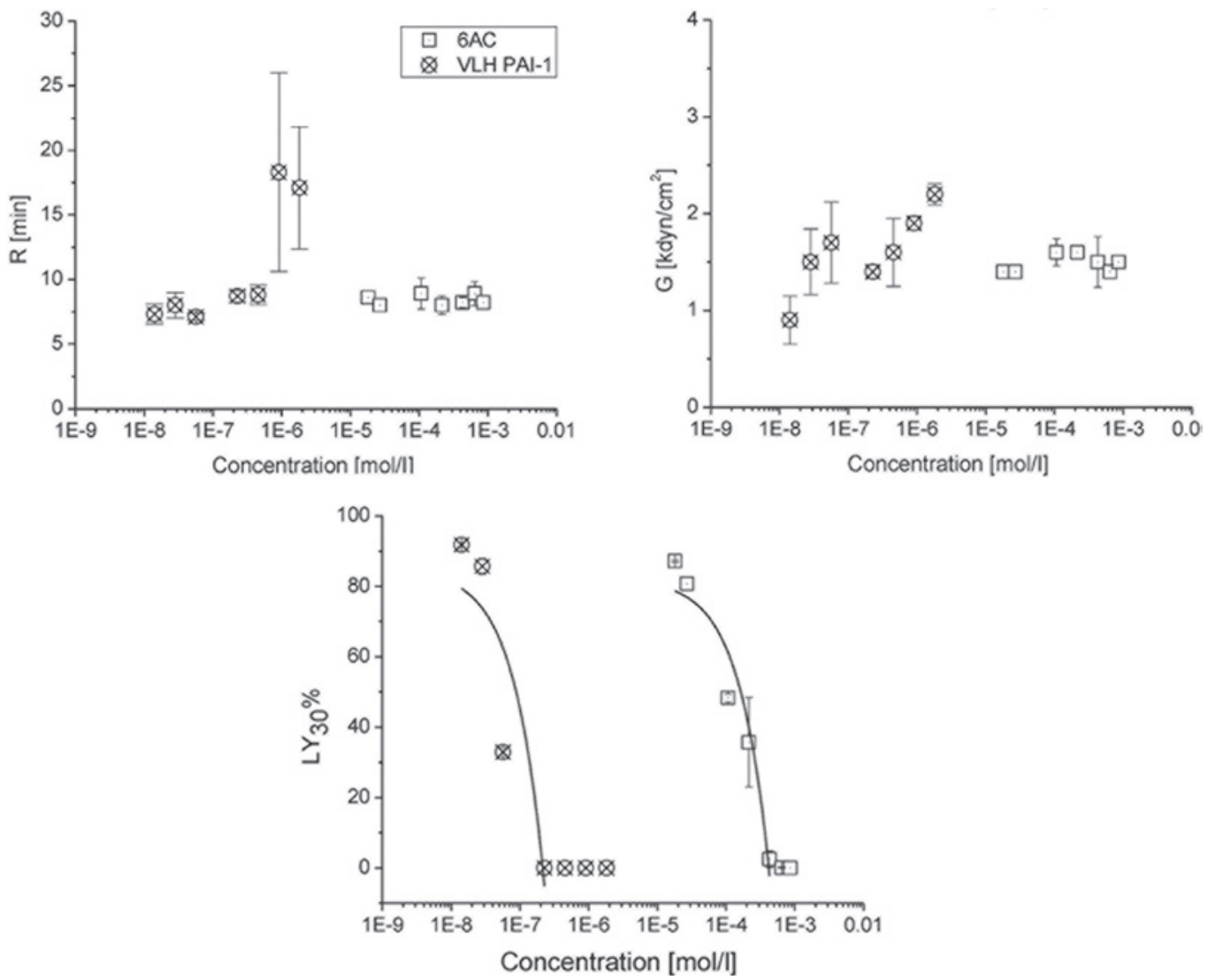

Figure 1. Effects of various concentrations of VLHL PAI-1 (circles) and 6AC (squares) on the R, G and $\mathrm{LY}_{30}$ values. IC $\mathrm{C}_{50}$ values (half maximal inhibitory concentrations) were calculated from the linear fit of four points for VLHL PAI-1 $\left(\mathrm{IC}_{50}=8.8 \times 10^{-8} \mathrm{~mol} / \mathrm{l}\right)$ and the linear fit of five points for $6 \mathrm{AC}\left(\mathrm{IC}_{50}=1.6 \times 10^{-4} \mathrm{~mol} / \mathrm{l}\right)$. $\mathrm{R}$, reaction time; $\mathrm{G}$, strength of clot; $\mathrm{LY}_{30}$, clot lysis 30 min after the maximum amplitude; 6AC, 6-aminocaproic acid; VLHL PAI-1, very long half life plasminogen activator inhibitor-1.

A

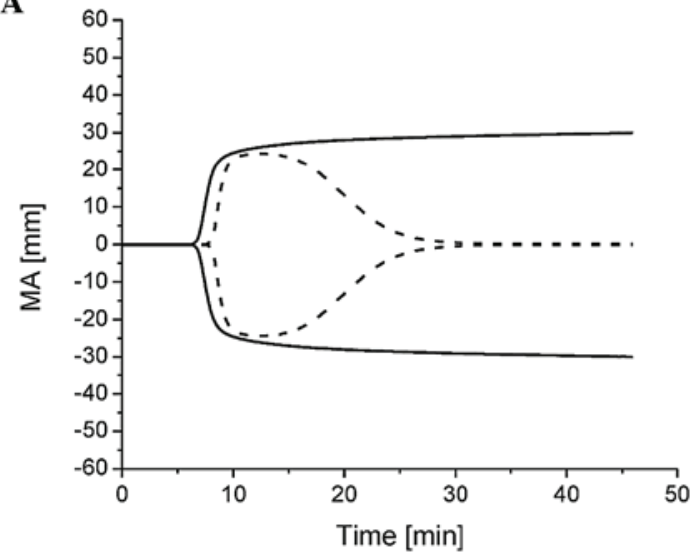

B

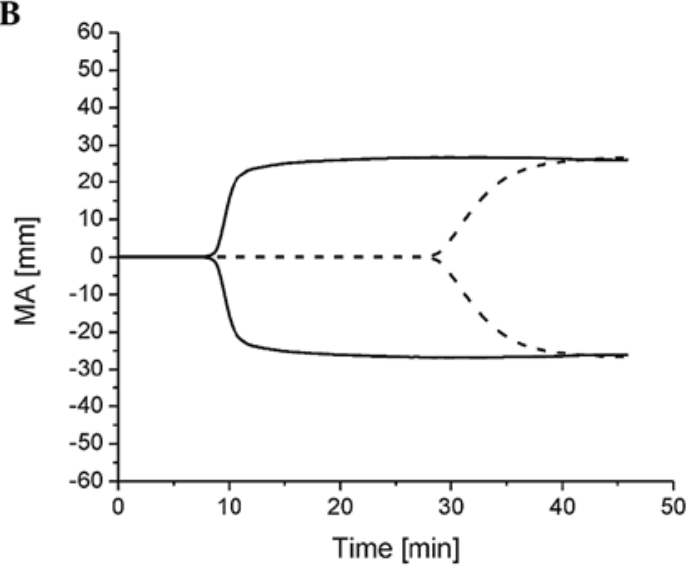

Figure 2. (A) Reference plasma clotted with kaolin and calcium alone (solid line) and with kaolin, calcium and tissue plasminogen activator (tPA; dotted line). Dotted line shows that the clot was quickly dissolved by tPA-activated plasmin. (B) Reference plasma clotted with kaolin, calcium, tPA and very long half life plasminogen activator inhibitor-1 (VLHL PAI-1) and with kaolin, calcium, tPA and a high concentration of VLHL PAI-1 (dotted line). Solid line shows that the addition of VLHL PAI-1 protected the clot from lysis, while the dotted line shows that increased concentrations of VLHL PAI-1 protected the clot from lysis, but delayed clot formation (reduced time from reaction until a 20 -mm clot formed) and reduced the angle of clot formation, indicating decreased fibrin cross-linking (reduced A). A, angle is the rate of clot development indicated by fibrinogen and fibrin cross-linking.

parameters for reference plasma in the literature. However, normal blood samples are considered to have a reaction time (R) of 2-8 min, a 2-4-min period between the reaction onset and the formation of a 20-mm clot, an MA of 51-69 mm and a $\mathrm{LY}_{30}$ value of $0-8 \%$ (12). 6-aminocaproic acid treatment decreases the incidence rate of clot lysis after $30 \mathrm{~min}$. Doses of 6-aminocaproic acid that were $\geq 18 \mu \mathrm{g}$ generated $\mathrm{LY}_{30}$ values of $\leq 5 \%$, indicating that the formed fibrin clot was almost entirely intact. Serial dilutions of $\leq 9 \mu \mathrm{g}$ yielded significantly different $(\mathrm{P}<0.01) \mathrm{LY}_{30}$ values, ranging 
between 26.7 and $87.4 \%$, as compared with the controls (Fig. 1). The $\mathrm{IC}_{50}$ of 6 -aminocaproic acid was $6.5 \mu \mathrm{g}$ or $1.6 \times 10^{-4} \mathrm{~mol} / 1$ when expressed as a concentration. No dosage of 6-aminocaproic acid produced a delay in clot formation (Fig. 1). The maximum $\mathrm{G}$ achieved by 6-aminocaproic acid was $1.8 \mathrm{kdyn} / \mathrm{cm}^{2}$.

VLHL PAI-1 treatment decreases incidence rate of clot lysis after $30 \mathrm{~min}$. At doses of $\geq 3.125 \mu \mathrm{g}$ VLHL PAI-1, tPA action was completely inhibited, generating $\mathrm{LY}_{30}$ values of $0 \%$. Doses of $\leq 0.78 \mu \mathrm{g}$ generated statistically significant differences $(\mathrm{P}<0.01)$ in $\mathrm{LY}_{30}$ values, which ranged between 32.1 and $92.2 \%$, as compared with the controls. The $\mathrm{IC}_{50}$ of VLHL PAI-1 was $0.68 \mu \mathrm{g}$ or $8.8 \times 10^{-8} \mathrm{~mol} / 1$ when expressed as a concentration (Fig. 1). In addition, VLHL PAI-1 doses of $\geq 12.5 \mu \mathrm{g}$ produced a statistically significant $(\mathrm{P}<0.01)$ increase in $\mathrm{R}$, as compared with the controls (Fig. 2). VLHL PAI-1 achieved a maximum $\mathrm{G}$ of $2.3 \mathrm{kdyn} / \mathrm{cm}^{2}$.

\section{Discussion}

There are three conformational states of PAI-1, including latent (inactive), active and reactive-center-cleaved (plasminogen-activator-complex) $(9,10)$. The active form of PAI-1 is rapidly converted (half-life, 1-2 h) into the latent form at a physiological temperature and $\mathrm{pH}(7,9,10,20)$. The reactive center loop of PAI-1, which contains the active site, is connected to $\beta$-sheet $\mathrm{C}$ at the $\mathrm{C}$-terminal and $\beta$-sheet $\mathrm{A}$ at the $\mathrm{N}$ terminal (9). In the latent form, the reactive center loop is inserted into $\beta$-sheet $A$ (central $\beta$-sheet), thereby eliminating the possibility of proteinase interaction with the reactive peptide bond (P1-P1') of tPA or UPA, and PAI-1 becomes inactive $(7,9,10)$. Thus, only active PAI-1 can be used as a clot protector. We previously developed a VLHL PAI-1 mutant that remained active for $>700 \mathrm{~h}$, and despite the two cysteine mutations, the VLHL PAI-1 structure was found to be almost identical to wild-type PAI-1 $(10,11,21,22)$. The long half-life of VLHL PAI-1 has enabled this protein to become a potential therapeutic agent for the mitigation of bleeding.

None of the current antifibrinolytics, including aprotinin, 6-aminocaproic acid and tranexamic acid (TXA), are without side effects, warranting the development of new hemostatic agents (14). TXA has been shown to have a statistical association with seizure induction, possibly due to cerebral ischemia caused by a decrease in regional or global cerebral blood flow (20). Aprotinin functions via the same mechanism as PAI-1, while lysine analogs, such as 6-aminocaproic acid and TXA, block the lysine-binding sites on plasminogen, thereby preventing the activation of plasmin and fibrin clot-degradation (19). The USA Food and Drug Administration has removed aprotinin, a bovine product, from the market due to statistical associations with renal failure, cerebrovascular events and mortality, without significantly stronger antifibrinolytic effects over other medications within the class (23-25). As a natural constituent of blood, VLHL PAI-1 may be free of side effects in addition to being an efficient antifibrinolytic agent.

Using a number of in vitro, ex vivo and in vivo models, previous studies have shown that VLHL PAI-1 is able to reduce clot lysis and bleeding in animal experiments (12-14,17). However, the efficacy of this protein had not been compared with other antifibrinolytics. The present study showed that VLHL PAI-1 is a more effective blood clot-protector than 6-aminocaproic acid. Racanelli et al compared recombinant wild PAI-1 to 6-aminocaproic acid in a rabbit model and found that the molar $\mathrm{ED}_{50}$ (effective dose for $50 \%$ of population base) was 25,000 times higher for 6-aminocaproic acid than for PAI-1. It was also found that complete inhibition of blood loss was achieved with PAI-1, but the highest dose of 6-aminocaproic acid did not completely inhibit blood loss (26). This outcome supports the conclusions of the present study. However, the absence of platelets and associated platelet function in reference plasma makes the clot weaker. This lack of thrombocytes in the reference plasma may cause 6-aminocaproic acid to exhibit an $\mathrm{ED}_{50}$ value 2,000 times higher than that of VLHL PAI-1.

Notably, an increase in $\mathrm{R}$ was observed in $\geq 12.5 \mu \mathrm{g}$ VLHL PAI-1 $\left(9.0 \times 10^{-7} \mathrm{~mol} / \mathrm{l}\right)$ cases. This phenomenon may be explained by the interaction of thrombin with PAI-1 and the simultaneous formation of cleaved PAI-1 and thrombin-PAI-1 complexes. The kinetics of this reaction are described by a suicide substrate model with a branched reaction that ends in the inhibitor/enzyme complex, the cleaved inhibitor and free enzyme. Due to the branched pathway, it was proposed that 3 mol PAI-1 was required to completely inhibit $1 \mathrm{~mol}$ thrombin (27). Since thrombin, a serine protease, converts soluble fibrinogen into insoluble strands of fibrin indirectly through factor XIII, inhibition of thrombin by excess PAI-1 increases the time required for clot formation.

VLHL PAI-1 may also be implemented as a treatment for PAI-1 deficiency. This condition is caused by a lack of PAI-1, an abnormality in the PAI-1 molecule itself, or defects in the secretory dynamics of PAI-1 in the blood (16,28-31). PAI-1 deficiency, a clinically rare bleeding disorder, is characterized by hyperfibrinolytic hemorrhage in the presence of normal thrombus formation $(7,9)$. Patients experience multiple episodes of uncontrolled bleeding, and in severe cases, require blood product transfusions (16,28-31). The class of medications currently used to treat PAI-1 deficiency are antifibrinolytics, which function by inhibiting the conversion of plasminogen into plasmin (22). There are three primary antifibrinolytics utilized in the clinical treatment of the disease: Aprotinin, 6-aminocaproic acid and TXA, which are not always effective and may exhibit a number of side effects (26). Bleeding occurs due to the unopposed conversion of plasminogen to plasmin by the action of tPA and subsequent fibrinolytic activity. Infusion of VLHL PAI-1, structurally homologous to wild-type PAI-1, may provide prophylactic treatment for these patients and restore them to normal health $(12-14,28,30)$.

In conclusion, the inhibition of tPA by VLHL PAI-1 demonstrates an improved efficacy over 6-aminocaproic acid in managing hemorrhagic events in the general patient population. However, concentrations of $>9.0 \times 10^{-7} \mathrm{~mol} / 1$ VLHL PAI-1 may delay the initiation and dynamics of clot formation. Therefore, in therapy, VLHL PAI-1 should be used in concentrations $<9.0 \times 10^{-7} \mathrm{~mol} / 1$.

\section{Acknowledgements}

This study was supported in part by grants from the Frank Stranahan Endowed Chair and Children's Miracle Network. 


\section{References}

1. Antun AG, Gleason S, Arellano M, et al: Epsilon aminocaproic acid prevents bleeding in severely thrombocytopenic patients with hematological malignancies. Cancer 119: 3784-3787, 2013.

2. Dhir A: Antifibrinolytics in cardiac surgery. Ann Card Anaesth 16: 117-125, 2013.

3. Jankun J and Skrzypczak-Jankun E: Plasminogen activator inhibitor with very long half-life (VLHL PAI-1) can reduce bleeding in PAI-1-deficient patients. Cardiovasc Hematol Disord Drug Targets 13: 144-150, 2013.

4. Mosesson MW: Fibrinogen and fibrin structure and functions. J Thromb Haemost 3: 1894-1904, 2005.

5. Rau JC, Beaulieu LM, Huntington JA and Church FC: Serpins in thrombosis, hemostasis and fibrinolysis. J Thromb Haemost 5 (Suppl 1): 102-115, 2007.

6. Zorio E, Gilabert-Estellés J, España F, Ramón LA, Cosín R and Estellés A: Fibrinolysis: the key to new pathogenetic mechanisms. Curr Med Chem 15: 923-929, 2008

7. Dellas C and Loskutoff DJ: Historical analysis of PAI-1 from its discovery to its potential role in cell motility and disease. Thromb Haemost 93: 631-640, 2005.

8. Cesarman-Maus G and Hajjar KA: Molecular mechanisms of fibrinolysis. Br J Haematol 129: 307-321, 2005.

9. Egelund R, Schousboe SL, Sottrup-Jensen L, Rodenburg KW and Andreasen PA: Type-1 plasminogen-activator inhibitor - conformational differences between latent, active, reactive-centre-cleaved and plasminogen-activator-complexed forms, as probed by proteolytic susceptibility. Eur J Biochem 248: 775-785, 1997.

10. Jankun J, Yang J, Zheng H, Han FQ, Al-Senaidy A and Skrzypczak-Jankun E: Remarkable extension of PAI-1 half-life surprisingly brings no changes to its structure. Int J Mol Med 29: 61-64, 2012.

11. Chorostowska-Wynimko J, Swiercz R, Skrzypczak-Jankun E, Wojtowicz A, Selman SH and Jankun J: A novel form of the plasminogen activator inhibitor created by cysteine mutations extends its half-life: relevance to cancer and angiogenesis. Mol Cancer Ther 2: 19-28, 2003.

12. Jankun J, Aleem AM, Selman SH, et al: Highly stable plasminogen activator inhibitor type one (VLHL PAI-1) protects fibrin clots from tissue plasminogen activator-mediated fibrinolysis. Int J Mol Med 20: 683-687, 2007.

13. Jankun J, Keck R, Selman SH and Skrzypczak-Jankun E: Systemic or topical application of plasminogen activator inhibitor with extended half-life (VLHL PAI-1) reduces bleeding time and total blood loss. Int J Mol Med 26: 501-504, 2010.

14. Jankun J, Selman SH, Keck RW, Łysiak-Szydłowska W and Skrzypczak-Jankun E: Very long half-life plasminogen activator inhibitor type 1 reduces bleeding in a mouse model. BJU Int 105: $1469-1476,2010$.

15. Jankun J, Skotnicka M, Łysiak-Szydłowska W, Al-Senaidy A and Skrzypczak-Jankun E: Diverse inhibition of plasminogen activator inhibitor type 1 by theaflavins of black tea. Int J Mol Med 27: 525-529, 2011

16. Jankun J and Skrzypczak-Jankun E: Yin and yang of the plasminogen activator inhibitor. Pol Arch Med Wewn 119: 410-417, 2009.
17. Jankun J, Aleem AM, Struniawski R, Lysiak-Szydłowska W, Selman SH and Skrzypczak-Jankun E: Accelerated thrombus lysis in the blood of plasminogen activator inhibitor deficient mice is inhibited by PAI-1 with a very long half-life. Pharmacol Rep 61: 673-680, 2009.

18. Morimoto Y, Yoshioka A, Imai Y, Takahashi Y, Minowa H and Kirita T: Haemostatic management of intraoral bleeding in patients with congenital deficiency of alpha2-plasmin inhibitor or plasminogen activator inhibitor-1. Haemophilia 10: 669-674, 2004.

19. Schulman S: Pharmacologic tools to reduce bleeding in surgery. Hematology Am Soc Hematol Educ Program 2012: 517-521, 2012.

20. Makhija N, Sarupria A, Kumar Choudhary S, Das S, Lakshmy R and Kiran U: Comparison of epsilon aminocaproic acid and tranexamic acid in thoracic aortic surgery: clinical efficacy and safety. J Cardiothorac Vasc Anesth 27: 1201-1207, 2013.

21. Harrop SJ, Jankova L, Coles M, et al: The crystal structure of plasminogen activator inhibitor 2 at 2.0 A resolution: implications for serpin function. Structure 7: 43-54, 1999.

22. Sharp AM, Stein PE, Pannu NS, et al: The active conformation of plasminogen activator inhibitor 1, a target for drugs to control fibrinolysis and cell adhesion. Structure 7: 111-118, 1999.

23. Fergusson DA, Hébert PC, Mazer CD, et al; BART Investigators: A comparison of aprotinin and lysine analogues in high-risk cardiac surgery. N Engl J Med 358: 2319-2331, 2008.

24. Howell N, Senanayake E, Freemantle N and Pagano D: Putting the record straight on aprotinin as safe and effective: results from a mixed treatment meta-analysis of trials of aprotinin. J Thorac Cardiovasc Surg 145: 234-240, 2013.

25. Iribarren JL, Jimenez JJ, Hernández D, et al: Postoperative bleeding in cardiac surgery: the role of tranexamic acid in patients homozygous for the $5 \mathrm{G}$ polymorphism of the plasminogen activator inhibitor-1 gene. Anesthesiology 108: 596-602, 2008.

26. Racanelli AL, Diemer MJ, Dobies AC, Dubin JR and Reilly TM: Comparison of recombinant plasminogen activator inhibitor-1 and epsilon amino caproic acid in a hemorrhagic rabbit model. Thromb Haemost 67: 692-696, 1992.

27. van Meijer M, Smilde A, Tans G, Nesheim ME, Pannekoek H and Horrevoets AJ: The suicide substrate reaction between plasminogen activator inhibitor 1 and thrombin is regulated by the cofactors vitronectin and heparin. Blood 90: 1874-1882, 1997.

28. Fay WP, Parker AC, Condrey LR and Shapiro AD: Human plasminogen activator inhibitor-1 (PAI-1) deficiency: characterization of a large kindred with a null mutation in the PAI-1 gene. Blood 90: 204-208, 1997.

29. Jankun J and Skrzypczak-Jankun E: Bleeding diathesis is associated with an A15T heterozygous mutation in exon 2 of the plasminogen activator inhibitor type 1. Exp Ther Med 1: 575-577, 2010.

30. Mehta R and Shapiro AD: Plasminogen activator inhibitor type 1 deficiency. Haemophilia 14: 1255-1260, 2008.

31. Schleef RR, Higgins DL, Pillemer E and Levitt LJ: Bleeding diathesis due to decreased functional activity of type 1 plasminogen activator inhibitor. J Clin Invest 83: 1747-1752, 1989.

32. Genét GF, Ostrowski SR, Sørensen AM and Johansson PI: Detection of tPA-induced hyperfibrinolysis in whole blood by RapidTEG, KaolinTEG, and functional fibrinogenTEG in healthy individuals. Clin Appl Thromb Hemost 18: 638-644, 2012. 